\title{
An Audit of a Hospital-Based Doppler Ultrasound Quality Control Protocol Using a Commercial String Doppler Phantom
}

\author{
S. Cournane \\ St. James's Hospital Dublin
}

A. J. Fagan

Centre for advanced medical imaging, St. James's hospital

Jacinta Browne

Technological University Dublin, jacinta.browne@tudublin.ie

Follow this and additional works at: https://arrow.tudublin.ie/scschphyart

Part of the Atomic, Molecular and Optical Physics Commons

\section{Recommended Citation}

Cournane, S., Fagan, A. J. and Browne, J.E. An Audit of a Hospital-Based Doppler Ultrasound Quality Control Protocol Using a Commercial String Doppler Phantom. Physica medica, 30 (2014) 380-384. doi:10.1016/j.ejmp.2013.10.001

This Article is brought to you for free and open access by the School of Physics \& Clinical \& Optometric Science at ARROW@TU Dublin. It has been accepted for inclusion in Articles by an authorized administrator of ARROW@TU Dublin. For more information, please contact arrow.admin@tudublin.ie, aisling.coyne@tudublin.ie, gerard.connolly@tudublin.ie.

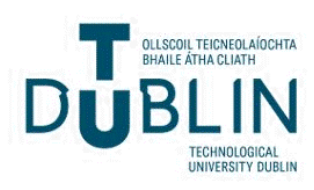




\title{
An Audit of a hospital-based Doppler ultrasound quality control protocol using a commercial string Doppler phantom
}

\author{
S. Cournane ${ }^{1}$, A. J. Fagan ${ }^{2}$ and J. E. Browne ${ }^{3}$ \\ ${ }^{1}$ Medical Physics and Bioengineering Department, St James's Hospital, Dublin, Ireland. \\ ${ }^{2}$ Centre for Advanced Medical Imaging (CAMI), St James's Hospital / Trinity College Dublin, Ireland. \\ ${ }^{3}$ IEO, School of Physics and FOCAS, Dublin Institute of Technology, Dublin, Ireland.
}

Keywords: Ultrasound, Doppler, Quality Assurance, String phantom

\begin{abstract}
:
Results from a four-year audit of a Doppler quality assurance (QA) program using a commercially available Doppler string phantom are presented. The suitability of the phantom was firstly determined and modifications were made to improve the reliability and quality of the measurements. QA of Doppler ultrasound equipment is very important as data obtained from these systems is used in patient management. It was found that if the braided-silk filament of the Doppler phantom was exchanged with an O-ring rubber filament and the velocity range below $50 \mathrm{~cm} / \mathrm{s}$ was avoided for Doppler quality control (QC) measurements, then the Maximum Velocity Accuracy (MVA) error and Intrinsic Spectral Broadening (ISB) results obtained using this device had a repeatability of $18 \pm 3.3 \%$ and $19 \pm 3.5 \%$, respectively. A consistent overestimation of the MVA of between $12 \%$ and $56 \%$ was found for each of the tested ultrasound systems. Of more concern was the variation of the overestimation within each respective transducer category: MVA errors of the linear, curvilinear and phased array probes were in the range $12.3-20.8 \%, 32.3-53.8 \%$ and $27-$ $40.7 \%$, respectively. There is a dearth of QA data for Doppler Ultrasound; it would be beneficial if a multicentre longitudinal study was carried out using the same Doppler ultrasound test object to evaluate sensitivity to deterioration in performance measurements.
\end{abstract}

\section{Introduction}

Diagnostic ultrasound imaging systems are routinely used to perform blood flow velocity measurements, via the Doppler effect, which assist in the clinical assessment and diagnosis of vascular and cardiac disease ${ }^{1,2}$. Doppler measurements of peak velocities carried out in the heart may be used to derive pressure gradients across the cardiac valve ${ }^{1}$, while blood flow for vascular applications provide an indication of stenosis in diseased vessels ${ }^{3}$. It is imperative that these ultrasound systems are capable of accurately measuring blood flow velocities to 
ensure correct diagnosis and appropriate patient treatment; however, previous studies have reported an overestimation in measurements of the maximum velocity by up to $40 \%$ in some systems ${ }^{4}$. This has been attributed to geometric spectral broadening effects, which are related to the active Doppler aperture size of the transducer and the angle correction used ${ }^{5,6}$.

It is thus surprising that little data has been published describing experience with Doppler QC testing in hospital environments. This may be due to the complex set-up and measure procedures using Doppler flow phantoms, which comprise blood mimicking fluid (BMF) pumped through vessels in tissue mimicking phantoms, often with various physiological flow profiles $^{7}$. Such systems are not easily portable and produce a limited range of velocities. Thus, focus is shifting towards the use of string phantoms, whose more simplified design may prove more suitable for the hospital environment. This is reflected in the 2010 report on QA of ultrasound imaging systems from the Institute of Physics and Engineering in Medicine (IPEM), $\mathrm{UK}^{8}$, wherein a string Doppler phantom was recommended for annual QC testing. Although string phantoms do not mimic the spread of blood flow velocities in a vessel they do provide a means of assessing an ultrasound system's absolute accuracy presenting a constant single velocity target. The spread in measured velocities may thus be attributed to the active aperture of the transducer.

Several string phantom designs have been described in the literature, each with particular strengths and weaknesses ${ }^{6,9,10}$ although there is currently only one commercially-available String phantoms: the CIRS Inc (Norfolk, USA) Model 043 Doppler string phantom. This phantom may be used with deionised water, obviating the need to prepare a speed-of-sound mixture of water and glycerol, thus increasing its attractiveness for a hospital environment. The phantom, however, has certain design features, specifically its braided-silk filament and wall-mounted motor, which are a cause for concern when incorporating it into a QC protocol $^{11}$. Previous studies have indicated back-scatter effects of silk filaments to be angulardependent, and effected by duration of immersion in fluid ${ }^{9}$. The wall-mounted motor may introduce vibrations along the filament and additionally, the effect of the string crossing the air-fluid interface may potentially be dragging air bubbles into the fluid, thus affecting string measurements ${ }^{8}$.

In any QC program, it is necessary to identify, characterise and eliminate any sources of error associated with a testing procedure so that tolerances may be established for use in tracking the performance of the system under test over time. The work presented in this paper is a critical evaluation of the CIRS string Doppler phantom and an audit of four years of a Doppler QA program using this test object. 


\section{Materials and Methods}

\section{Doppler string phantom}

The CIRS Model 043 Doppler string phantom consists of an open-topped tank, with a fivewheel pulley assembly arranged such that a braided-silk filament (diameter: $0.28 \pm 0.02 \mathrm{~mm}$ ) is driven by a motor shaft attached through the external tank wall and positioned above the fluid line. The filament velocity is controlled by an external controller which produces set velocities between $10-200 \mathrm{~cm} / \mathrm{s}$. An O-ring rubber filament (Radionics Ltd., Ireland), with diameter $1.6 \pm 0.02 \mathrm{~mm}$, similar to that described in previous studies was also used in this work $^{9,12}$. The tank was filled with degassed water of acoustic velocity $1482 \mathrm{~m} / \mathrm{s}$, maintained at a temperature of $22 \pm 1{ }^{\circ} \mathrm{C}$ and with a rubber mat placed at the Perspex tank bottom to absorb ultrasonic reflections. As detailed in the product literature, the phantom can compensate for the acoustic velocity of water by adjusting the string filament velocity and displaying the velocity measures by the ultrasound system using a calibrated acoustic velocity of $1540 \mathrm{~m} / \mathrm{s}$. The accuracy of the string velocities produced by the phantom were verified using a tachometer (model 465, Testo Ltd., UK).

\section{Test Procedure and Data Acquisition}

\section{Evaluation of CIRS String Phantom}

A Sonoline Antares (Siemens, Germany) ultrasound system with linear array transducer (VFX13-5) was used for the phantom evaluation. The transducer was held above the phantom using a retort stand and clamp, positioned at an incident angle of $60^{\circ}$ and depth of $43 \mathrm{~mm}$ from the filament. For all measurements, the angle correction cursor was aligned with the B-mode image of the filament at $60^{\circ}$ and a Pulsed-wave Doppler spectrum was acquired, as described in IPEM Report No. $102^{8}$. The MVA error and ISB measurements were calculated for set velocities $\left(V_{\text {set }}\right)$, using equations 1 and 2 ,

$\operatorname{MVA}(\%)=\left(\frac{V_{\max }}{V_{\text {set }}}-1\right) \times 100$

$$
I S B(\%)=\frac{V_{\max }-V_{\min }}{V_{\max }+V_{\min } \mathrm{II}} \times 100
$$

with four measurements of the maximum $\left(V_{\max }\right)$ and minimum $\left(V_{\min }\right)$ velocities, respectively, measured by placing the cursor at the peak and trough of the spectra, ignoring noise spikes, as 
shown in Figure 1. $V_{\max }$ and $V_{\min }$ measurements were examined in $10 \mathrm{~cm} / \mathrm{s}$ increments across the range $10-200 \mathrm{~cm} / \mathrm{s}$ for the brailed-silk filament, and where resonance effects were witnessed, between 20 to $50 \mathrm{~cm} / \mathrm{s}$, measurements were recorded in increments of $2 \mathrm{~cm} / \mathrm{s}$.

In the set velocity range $50-200 \mathrm{~cm} / \mathrm{s}$, measurements were repeated for the braided-silk filament at soak times of between 0 hours to 24 . In all cases, the standard deviation for the reported MVA error and ISB parameter values were determined by propagation of errors using the standard deviations of the individual measurements. A paired Student's t-test analysis was carried out to check for statistically significant differences $(p<0.05)$ in the MVA error and ISB for the different soak time datasets. The measurements were carried out initially for a braided-silk filament and then repeated with the O-ring filament in its place.

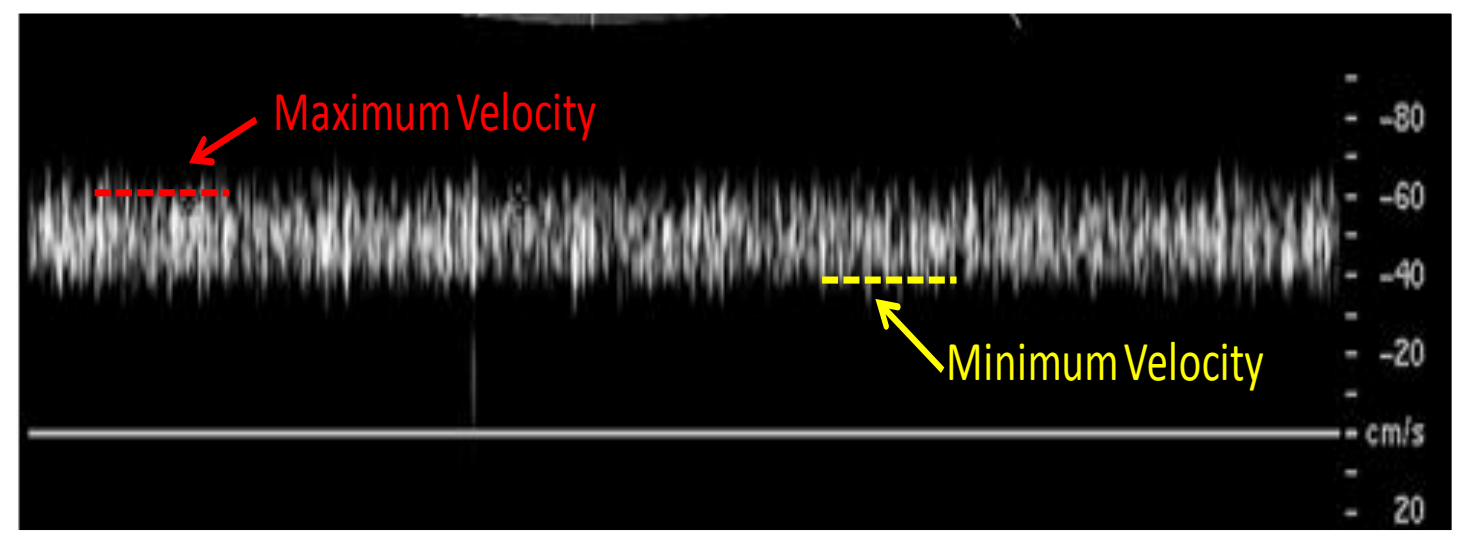

Figure 1: Measurements of the maximum $\left(V_{\max }\right)$ and minimum $\left(V_{\min }\right)$ velocities as performed on a Pulsed-wave Doppler spectrum.

The repeatability of the MVA error and ISB measurements using this String Phantom set-up was evaluated by obtaining eight sets of measurements for a set velocity of $50 \mathrm{~cm} / \mathrm{s}$, with the transducer repositioned over the filament between each measurement in order to mimic the change in QC set-up from year to year. These measurements were determined using both the phantom's default transducer clamping mechanism and a simple retort stand and clamp setup. The default clamp is extremely manoeuvrable, offering 6 degrees of freedom while the simple retort stand and clamp offer just 3 degrees of freedom.

To verify the accuracy of the phantom's acoustic velocity correction factor, MVA error and ISB measurements were carried out using two different speed-of-sound corrected solutions of degassed water with either $9.5 \%$ glycerol or $9 \%$ propanol per volume. Both of these solutions produced an acoustic velocity of $1540 \mathrm{~m} / \mathrm{s}$ at room temperature. 


\section{Audit of Doppler QA Program}

The modified phantom (with the O-ring filament in situ) was incorporated into a QA program in a busy hospital (St. James's Hospital, Dublin). Nine ultrasound systems in current clinical use, spanning the areas of peripheral and abdominal vascular, cardiology and radiology were tested using the recommended IPEM report No. 102 Doppler ultrasound QA protocol. The ultrasound systems comprised of the following mid- to high-end scanners: Vivid i and Vivid 7 (General Electric); Preirus (Hitachi); HDI5000, iU22, iE33 and Sonos 5500 (Philips); Sonoline Antares (Siemens); and Z.one (Zonare).Testing was carried as part of the commissioning protocol to establish baseline data. Five of the systems were tested annually thereafter, and that Doppler QA data is presented here as part of a four-year audit. Measurements were obtained for the MVA, ISB, range gate registration, sample volume dimensions, highest detectable velocity, and wall filter levels, with all measurements performed over the velocity range $50-200 \mathrm{~cm} / \mathrm{s}$. Baseline data was taken at commissioning with tolerances for each system calculated based on a propagation of errors combining the set-up variation due to the CIRS default clamp, the established standard deviation of the MVA and ISB metrics, and also the determined error on the set string velocity itself. For the longitudinal data, repeat measurements were performed using the same system protocol settings. For commissioning, the assessed transducers were grouped as follows: 1-5 were linear array transducers $(7-12 \mathrm{MHz})$ while those numbered 6-10 and 11-15 denote curvilinear $(3.5-6 \mathrm{MHz})$ and phased array transducers $(2-4 \mathrm{MHz})$, respectively.

\section{Results}

Measurements of the MVA error and ISB using the braided-silk filament soaked for 1 hour are presented in Figure 2. Above $50 \mathrm{~cm} / \mathrm{s}$, little variability was observed in either parameter; however, significant deviations from the average values were observed in the range $20-$ $46 \mathrm{~cm} / \mathrm{s}$ and thus additional measurements were taken. Similar patterns were observed in these velocity ranges for different soak times, suggesting a possible mechanical resonance within the phantom affecting measurements. Consequently, only measurements between 50 and $200 \mathrm{~cm} / \mathrm{s}$ were considered. 


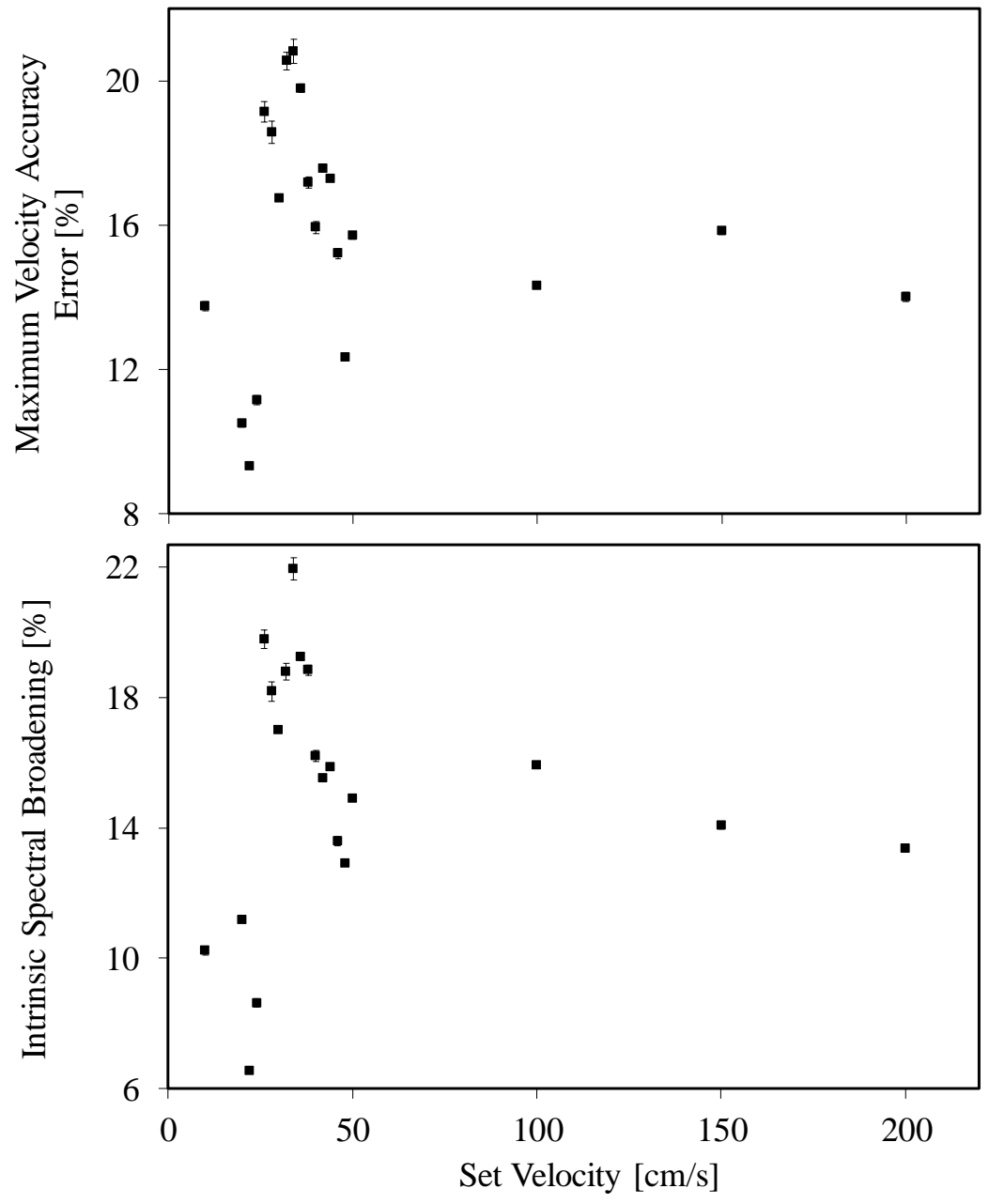

Figure 2: MVA error and ISB $( \pm \sigma)$ as a function of set velocity with a braided-silk filament after 1 hour soak time, illustrating a resonant-like behaviour below $50 \mathrm{~cm} / \mathrm{s}$.

The MVA error and ISB as a function of the braided-silk filament soak time for set velocities in the range $50-200 \mathrm{~cm} / \mathrm{s}$ are presented in Figure 3. The values for both parameters increased as a function of the soak time across the set velocities range. This increase was significant $(\mathrm{p}<0.05)$ after 3 hours of soaking time with mean increases of $24 \%$ and $20 \%$ for the MVA error and ISB, respectively, exhibited after 24 hours. Measurements performed using the O-ring filament revealed no significant variability with soak time. 


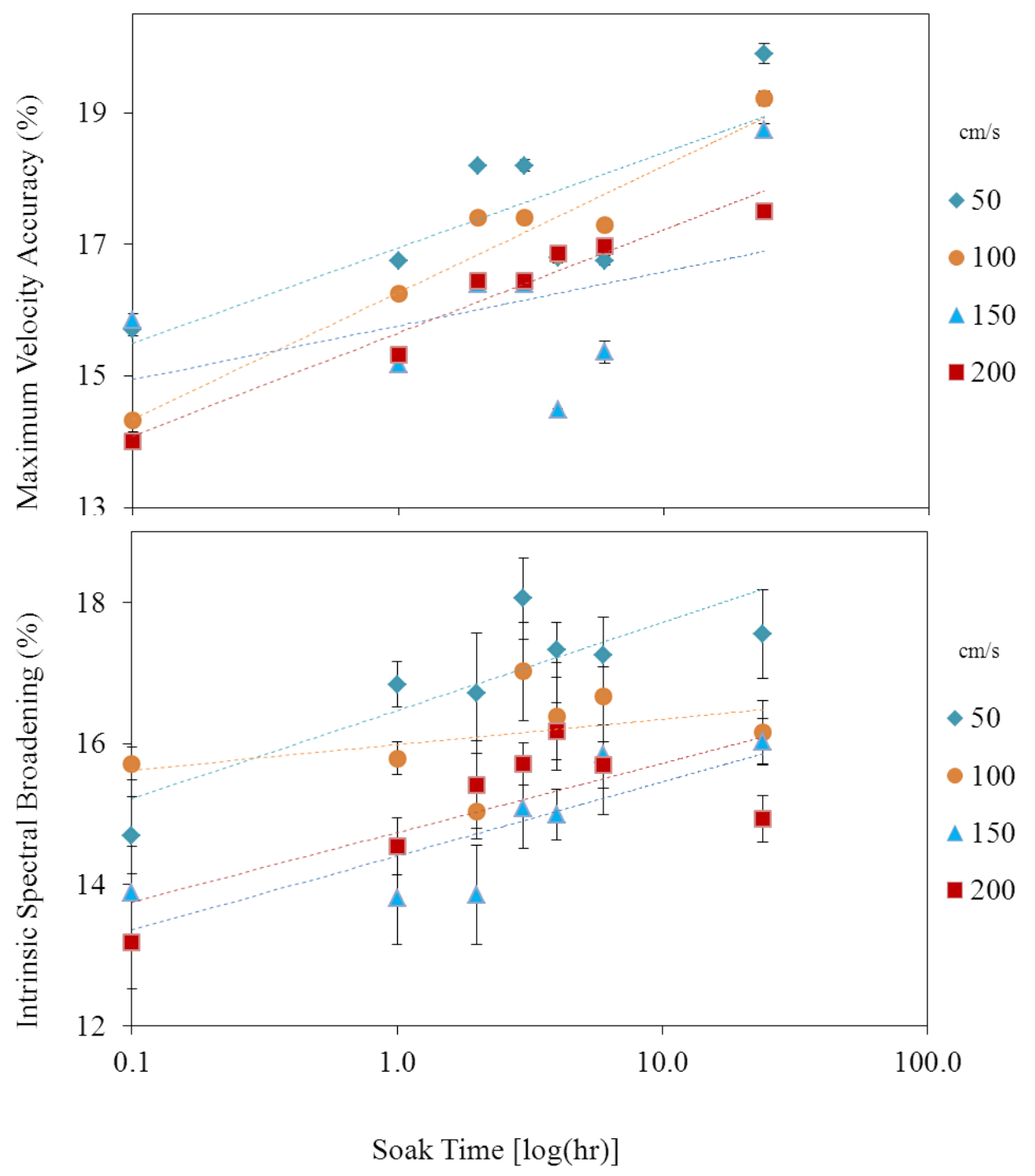

Figure 3: MVA error and ISB $( \pm \sigma)$ measured as a function of the braided-silk filament soak time. Dashed trend lines illustrate the increase in parameter values with increasing soak time.

Repeatability measurements of the MVA error and ISB at a set velocity of $50 \mathrm{~cm} / \mathrm{s}$, after repositioning of the set-up, averaged at $18 \pm 3.3 \%$ for the MVA error and $19 \pm 3.5 \%$ for the ISB. When replaced with the simple clamp and retort stand, the parameter values were found to reduce to $16 \pm 1.6 \%$ and $14 \pm 1 \%$, respectively. The calibration data for the phantom as measured using the tachometer revealed errors of less than $1.5 \%$ in the actual string velocity for velocities up to $200 \mathrm{~cm} / \mathrm{s}$. Furthermore, no significant difference was found between the measurements using the degassed water with the correction implemented by the string phantom, or the speed-of-sound-corrected solutions.

Figure 4 presents the MVA error and ISB for each transducer at commissioning testing. The data represents the measurements averaged over the set velocity range $50-200 \mathrm{~cm} / \mathrm{s}$, 
respectively, with error bars depicting the standard deviation. There was significant variation across all transducer types, especially for the curvilinear transducers where the MVA and ISB were in the range $18-46 \%$ and $22-56 \%$, respectively. In addition, measurements of the range gate registration, sample volume dimensions, highest detectable velocity and wall filter levels were carried out with no changes in performance detected for any of the transducers tested over the four-year period.

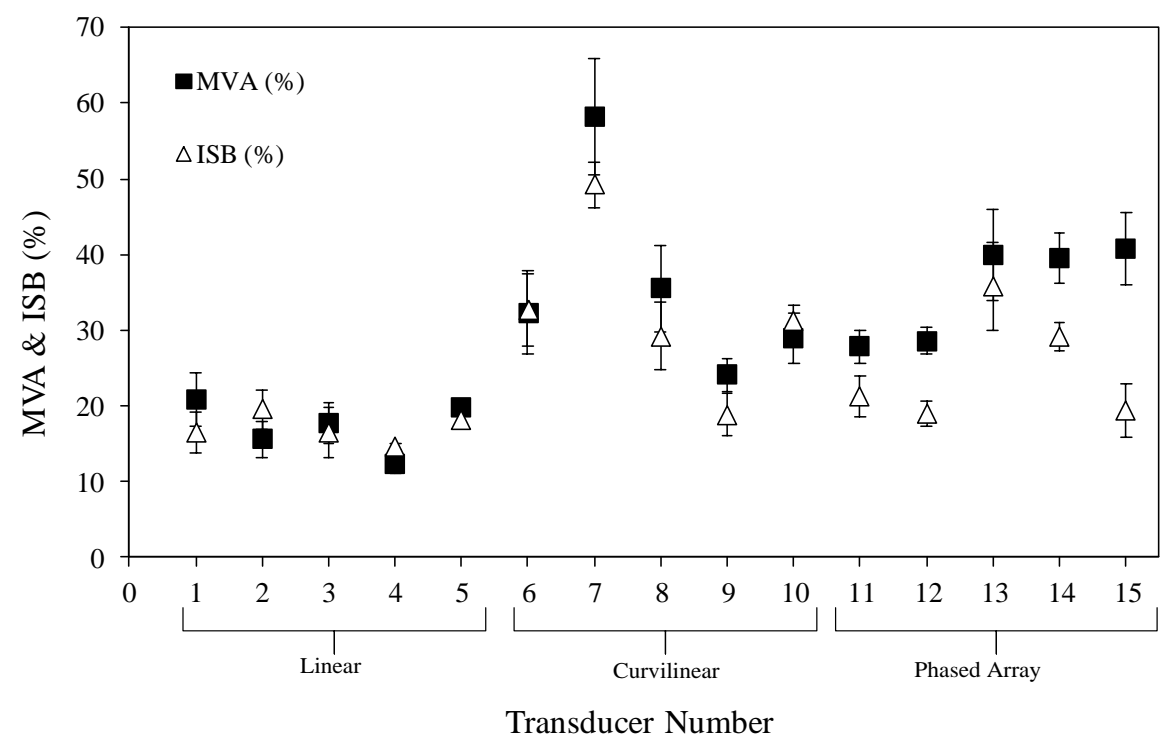

Figure 4 MVA error and ISB for a range of linear (1-5), curvilinear (6-10) and phased-array (11-15) transducers, as measured during commissioning. The data represents the average value $( \pm \sigma)$ for each probe measured across a set velocity range of $50-200 \mathrm{~cm} / \mathrm{s}$.

Longitudinal MVA error and ISB data for five clinical scanners included in the routine Doppler QA program over a four-year period are presented in Figure 5. Baseline tolerance limits for the MVA error and ISB were determined to be $\pm 19 \%$ and $\pm 20 \%$ of the baseline values, respectively, measured at commissioning. 


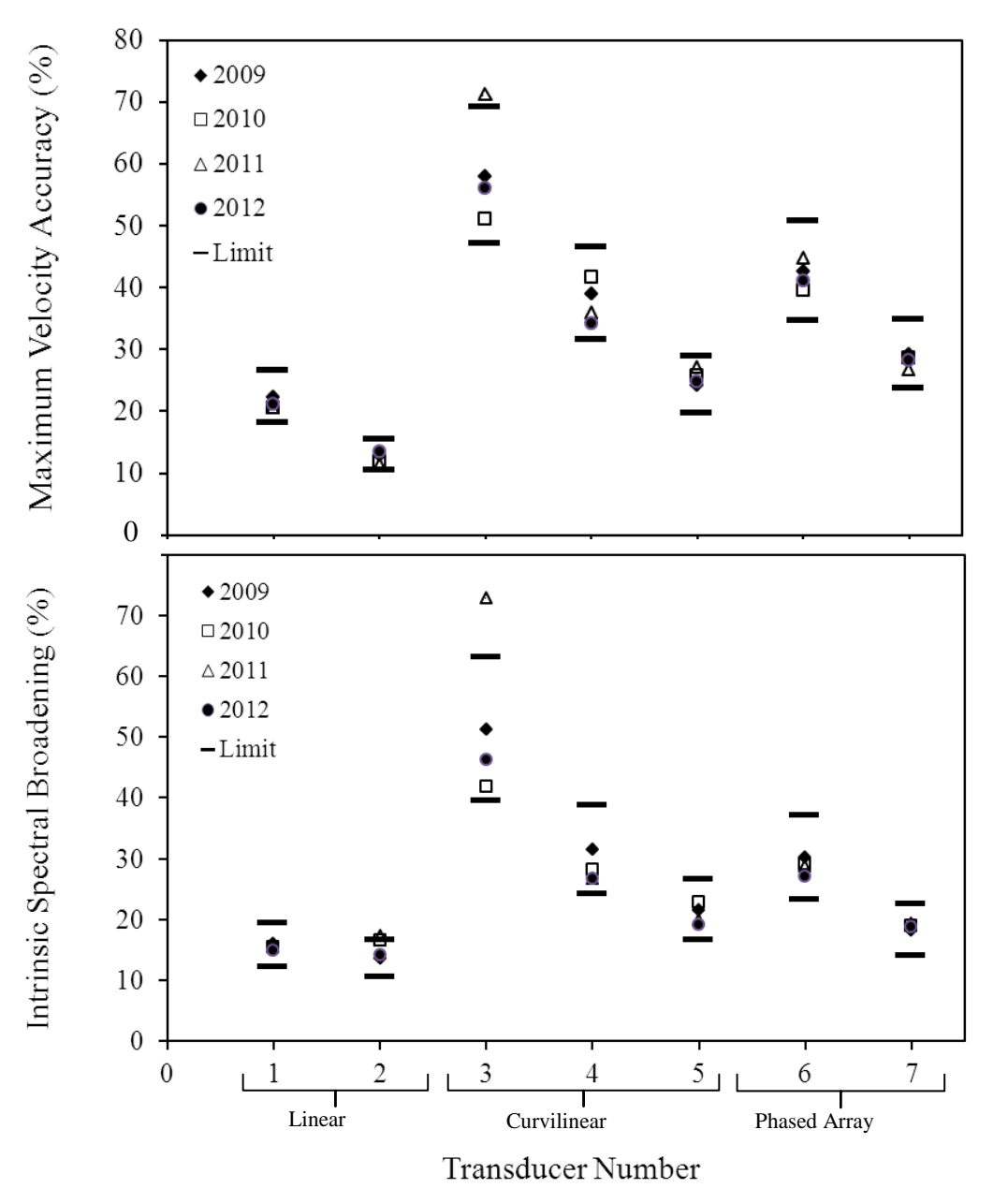

Figure 5: MVA error and ISB for a range of linear (1-2), curvilinear (3-5) and phased-array (6-7) transducers included in the longitudinal Doppler QA program. Measurements represent the average value $( \pm \sigma)$ over a set velocity range of $50-200 \mathrm{~cm} / \mathrm{s}$ with tolerance limits depicted as $\pm 19 \%$ and $\pm 20 \%$ for the MVA error and ISB, respectively.

It can be seen from Figure 5 that both parameters for transducer number 3 fell outside the tolerance level in 2011. The data for this transducer was interrogated further and is presented in Figure 6 as the yearly MVA error and ISB measurements over the set velocity range 50 $200 \mathrm{~cm} / \mathrm{s}$. The 2011 data shows the parameters drifting outside baseline levels, with the exception of MVA error for $50 \mathrm{~cm} / \mathrm{s}$ which approaches but does not exceed the limits. This increase in both the MVA error and the ISB was attributed to a number of damaged piezocrystals on the probe face, which led its removal from clinical service. Data from the replacement probe was included as the 2012 data in Figures 5 and 6. 


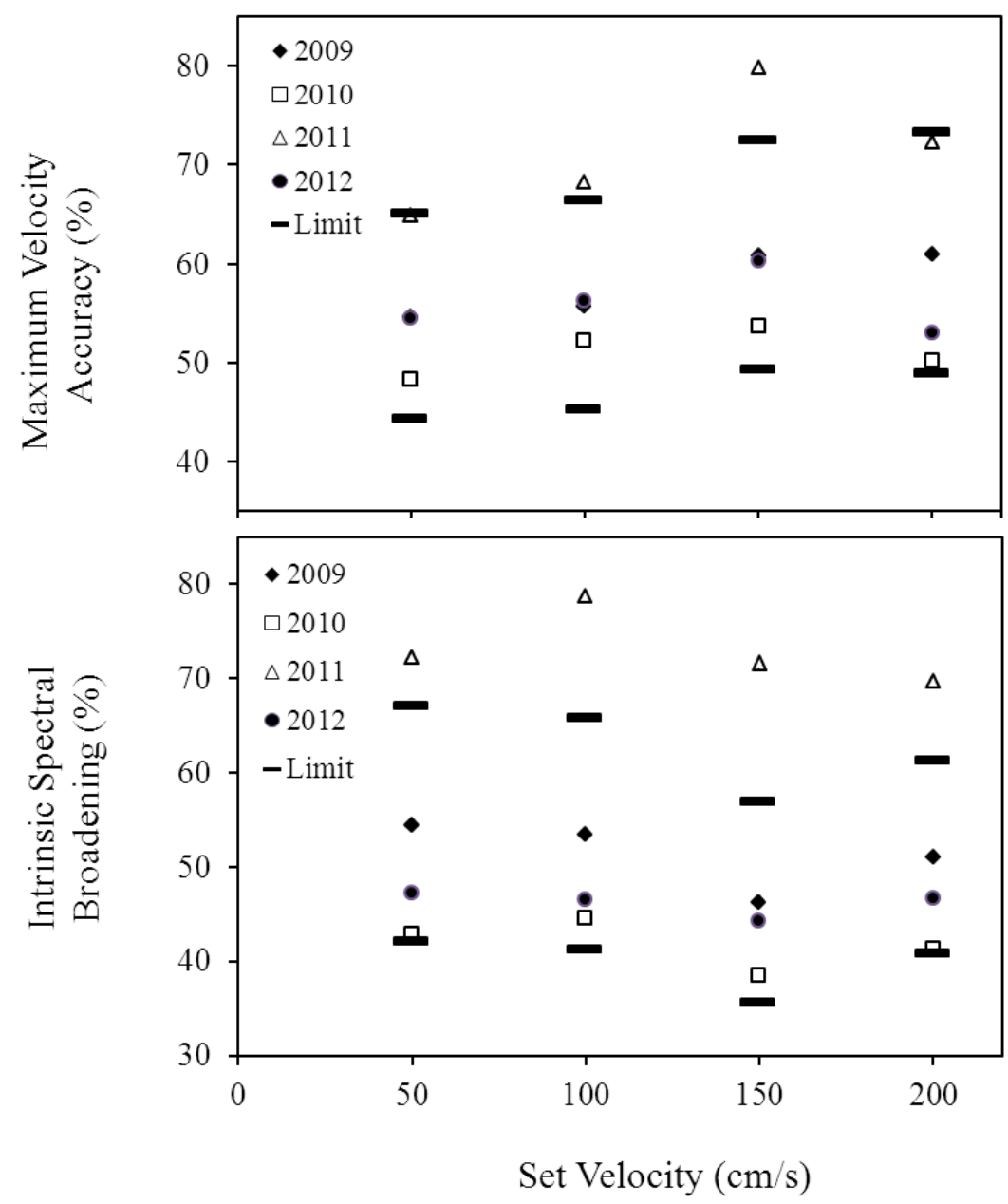

Figure 6: MVA error and ISB data as a function of set velocity for transducer number 3, measured over the four-year audit, indicating the problem revealed in the 2011 data. The tolerance limits depicted were set at $\pm 19 \%$ and $\pm 20 \%$ of the baseline MVA error and ISB,

respectively.

\section{Discussion}

A critical evaluation of the CIRS Doppler string phantom's suitability for use in a hospitalbased QA program was carried out. In a typical QA scenario, the string phantom would be set-up and used to test several ultrasound systems sequentiallyand hence the change in MVA error and ISB with the braided-silk filament over time is unacceptable. A simple replacement O-ring filament overcame this limitation, increasing the reproducibility of the measurements. The resonant-like variability observed in the set velocity range $20-50 \mathrm{~cm} / \mathrm{s}$ was attributed to vibrations deriving from the wall-mounted motor and, thus, this set velocity range should be avoided using this device. 
Calibration of the filament velocity, necessary for ensuring annual consistency of measurement, was performed using a tachometer; however, where such a device is not accessible the true filament velocity may be determined by dividing the string's length by the time measured for one filament revolution, as has been detailed previously ${ }^{6}$. A further improvement, to be implemented at the commissioning stage, would be to employ a simple retort stand instead of the CIRS clamp.

With these simple modifications applied, the phantom was employed as part of a commissioning and QA program. For each ultrasound transducer presented in Figure 4, a consistent overestimation of the set velocity was evident, reflecting similar findings from a previous study ${ }^{6}$, suggesting that little improvement has been made in the Doppler ultrasound maximum velocity measurement process implemented by ultrasound scanner manufacturers. Of more concern is the variation of the overestimation within each respective transducer category, where the MVA error of the linear, curvilinear and phased array probes were in the range $12.3-20.8 \%, 32.3-53.8 \%$ and $27.8-40.7 \%$, respectively. Such variations across transducers could potentially lead to a mis-categorisation of a stenosis. At a time when resources are being utilised to maximum capacity with an ever-increasing need to interchange scanners in busy clinics, this variation in performance is a concern for longitudinal patient management.

The importance of performing functionality testing at commissioning for radiology or gynaecology scanners should also be highlighted. The clinical applications of such systems may evolve over time should clinical evidence determine the value in utilising blood velocity measurements for diagnostic purposes in these clinical areas. Accordingly, it would be necessary to establish baseline data on these systems and, indeed, determine whether a system is fit for the new clinical application. For radiology ultrasound scanners, an important performance metric is sensitivity and penetration depth ${ }^{13}$. While this measurement has been carried out previously using a modified Doppler string phantoms with a castor oil medium ${ }^{14}$, commercially available phantoms are currently unsuitable for performing this measurement. This is an area that requires investigation to determine the value of carrying out sensitivity and penetration depth measurements for routine QA purposes.

As part of the current audit, annual QC testing for vascular and cardiology ultrasound scanners over a four-year period was reviewed. One curvilinear transducer (number 3 in Figure 5) was of most interest as damaged piezo-crystals had resulted in the MVA error and ISB performance of this transducer falling outside of tolerance. The MVA error and ISB both increased from baseline measurements of $58 \pm 3 \%$ and $51 \pm 3 \%$ to $71.3 \pm 5 \%$ and $73 \pm 6 \%$, 
respectively. IPEM Report 102 recommends Doppler measurements be taken at a set velocity of $50 \mathrm{~cm} / \mathrm{s}$; however in this case (Figure 6), measurements at $50 \mathrm{~cm} / \mathrm{s}$ resulted in the MVA error being affected by the resonant artefact of the motor vibrations. When examined as a function of set velocity, the change in the probe performance was outside tolerance for both parameters at set velocities of $100 \mathrm{~cm} / \mathrm{s}$ and $150 \mathrm{~cm} / \mathrm{s}$. In a QC program, it would thus be advisable to concentrate on the higher velocity of $100 \mathrm{~cm} / \mathrm{s}$ which is unaffected by the motor vibrations and within the reported physiologically relevant range, $1-100 \mathrm{~cm} / \mathrm{s}^{15}$.

In the case of the damaged transducer the Doppler ultrasound QC measurements provided a quantitative means of establishing that a defect had occurred in the transducer, leading to a significant increase of the MVA error and therefore, potentially affecting longitudinal patient management. However, the relatively simple crystal dropout QC test had prompted the performance of Doppler testing for confirmation of the defect. The results from the crystal dropout test alone would have provided sufficient evidence for the removal of this transducer from service which begs the question as to whether it is necessary to conduct Doppler QC tests on a regular basis, or rather to simply perform baseline commissioning with reactive tests only conducted when faults are suspected. However, the significant increase in the MVA error observed here suggests that Doppler QC measurements could offer a more sensitive measure of deterioration in the transducer performance compared with the crystal dropout test, particularly for cases where transducer delamination has occurred or a small number of elements have been damaged. Nevertheless, the fact remains that insufficient evidence currently exists in the form of a large scale longitudinal evaluation of Doppler systems' performance that correlates crystal damage or electronic board failure with its subsequent effect on Doppler velocity measurements and, therefore, on the value of carrying out routine Doppler QC testing.

\section{Conclusion}

This study presents an evaluation of the commercially available CIRS string phantom and its use in an ultrasound QC program. It was found that small changes could be made to the string phantom to improve its suitability for inclusion into a Doppler QA program. The Doppler MVA error and ISB for nine ultrasound scanners were presented showing that a significant overestimation still exists in the maximum velocity measurement. A particular worry is the variation of the overestimation within transducer categories which could potentially affect longitudinal patient management. Doppler QC measurements carried out over a four year period highlighted the importance of these measurements in assessing the effect of deterioration of a transducer, due to crystal dropout damage, on Doppler measurement performance. However, there is still insufficient longitudinal data available to determine the 
sensitivity of Doppler QC measurements to changes in transducer and ultrasound scanner performance over time, emphasising the need for the collation of an evidence-base of longitudinal data from a larger number of ultrasound systems in order to determine the role and value of this type of Doppler QC testing.

\section{References}

[1] Holen J, Aaslid R, Landmark K, Simonsen S. Determination of pressure gradient in mitral stenosis with a non-invasive ultrasound Doppler technique. Acta medica Scandinavica 1976;199(6):455-60.

[2] Thrush A, Hartshorne T. Peripheral Vascular Ultrasound: How, Why and When. Churchill Livingstone, 1999.

[3] Oates CP, Naylor AR, Hartshorne T, et al. Joint recommendations for reporting carotid ultrasound investigations in the United Kingdom. European journal of vascular and endovascular surgery : the official journal of the European Society for Vascular Surgery 2009;37(3):251-61.

[4] Winkler AJ, Wu J. Correction of intrinsic spectral broadening errors in Doppler peak velocity measurements made with phased sector and linear array transducers. Ultrasound in medicine \& biology 1995;21(8):1029-35.

[5] Hoskins PR. Estimation of blood velocity, volumetric flow and wall shear rate using Doppler ultrasound. Ultrasound in medicine \& biology 2011;19:120.

[6] Hoskins PR. Accuracy of maximum velocity estimates made using Doppler ultrasound systems. The British journal of radiology 1996;69(818):172-7.

[7] King DM, Moran CM, McNamara JD, Fagan AJ, Browne JE. Development of a vessel-mimicking material for use in anatomically realistic Doppler flow phantoms. Ultrasound in medicine \& biology 2011;37(5):813-26.

[8] Quality Assurance of Ultrasound Imaging Systems. In: Russel S, editor. Institute of Physics and Engineering in Medicine (IPEM) Report No 102, 2010

[9] Hoskins PR. Choice of moving target for a string phantom: I. Measurement of filament backscatter characteristics. Ultrasound in medicine \& biology 1994;20(8):773-80.

[10] Gittins J, Martin K. The leicester Doppler phantom--a digital electronic phantom for ultrasound pulsed Doppler system testing. Ultrasound in medicine \& biology 2010;36(4):647-55.

[11] Browne J, Cournane S, Fagan A. Evaluation of CIRS string Doppler phantom as a test tool for use in a Doppler Ultrasound Quality Assurance program. Physica Medica 2012;28(4):334.

[12] Hoskins PR. Choice of moving target for a string phantom: II. On the performance testing of Doppler ultrasound systems. Ultrasound in medicine \& biology 1994;20(8):781-9.

[13] Browne JE, Watson AJ, Hoskins PR, Eliott AT. Validation of a sensitivity performance index test protocol and evaluation of colour Doppler sensitivity for a range of ultrasound scanners. Ultrasound in medicine \&biology 2004; 30(11): 1475-83.

[14] Goldstein A. Performance tests of Doppler ultrasound equipment with a string phantom. J Ultrasound in Medicine 1991;10(3):125-39.

[15] AIUM (American Institute of Ultrasound in Medicine. Performance Criteria and Measurements for Doppler Ultrasound Devices. American Institute of Ultrasound in Medicine Standards Committee 2002. 